\section{ORIGINAL RESEARCH}

\section{A. Gupta}

R.J. Young

S. Karimi

S. Sood

Z. Zhang

Q. Mo

P.H. Gutin

A.I. Holodny

A.B. Lassman

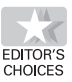

\title{
Isolated Diffusion Restriction Precedes the Development of Enhancing Tumor in a Subset of Patients with Glioblastoma
}

BACKGROUND AND PURPOSE: Most response criteria for patients with glioblastoma rely on increases in the contrast enhancing abnormality to determine tumor progression. Our aim was to determine retrospectively in patients with glioblastoma whether diffusion restriction can predict the development of new enhancing mass lesions.

\begin{abstract}
MATERIALS AND METHODS: We reviewed the brain MR imaging scans (including DWI and ADC maps) of 208 patients with glioblastoma. Patients with restricted diffusion in or adjacent to the tumor were identified, with further analysis only performed on those patients with low-ADC lesions without enhancement. These patients were followed to determine if new concordant enhancement developed at the site of the low-ADC lesion. A Wilcoxon signed rank test, competing risk analysis, and KaplanMeier curves were used to compare the mean drop in ADC values, assess enhancement-free survival, and determine overall survival, respectively.
\end{abstract}

RESULTS: In 67 of the 208 patients (32.2\%), visibly detectable restricted diffusion was seen during treatment. The study cohort was formed by the 27 patients with low-ADC lesions and no corresponding enhancement. Twenty-three (85.2\%) patients developed gadolinium-enhancing tumor at the site of restricted diffusion a median of 3.0 months later $(95 \% \mathrm{Cl}, 2.6-4.1$ months). The mean decrease in ADC was $22.9 \%$ from baseline $(P<.001)$. The 3-month enhancement-free survival probability was 0.481 (95\% Cl, 0.288-0.675). The 12-month overall survival probability was $0.521(95 \% \mathrm{Cl}, 0.345-0.788)$. Restricted diffusion predicted enhancement regardless of antiangiogenic therapy with bevacizumab.

CoNCLUSIONS: In a subset of patients with glioblastoma, development of a new focus of restricted diffusion during treatment may precede the development of new enhancing tumor.

ABBREVIATIONS: $\mathrm{ADC}=$ apparent diffusion coefficient; Cho = choline; $\mathrm{Cl}=$ confidence interval; $\mathrm{DCE}=$ dynamic contrast-enhanced; DSC = dynamic susceptibility contrast; DWI = diffusionweighted imaging; HIPPA = Health Insurance Portability and Accountability Act; HR = hazard ratio; $\mathrm{NAA}=\mathrm{N}$-acetylaspartate; $\mathrm{PET}=$ positron-emission tomography; $\mathrm{rCBV}=$ relative cerebral blood volume

G lioblastomas are the most malignant of the primary brain tumors. ${ }^{1}$ Change in contrast-enhancing abnormality on brain MR imaging is the current imaging standard for diagnosis, assessment of prognosis, and management of these tumors. Enhancing tumor may occasionally show areas of restricted diffusion (ie, concordant lesions). Although the causes for restricted diffusion are many, for malignant tumors such as glioblastomas, medulloblastomas, or lymphomas, the dominant factor contributing to tumor-related diffusion restriction is thought to be related to regions of increased cellularity. ${ }^{2-4}$ The significance of isolated restricted diffusion without enhancement (ie, discordant lesions) is less well understood. Although the role of pretreatment quantitative DWI has been

Received May 27, 2010; accepted after revision December 2.

From the Department of Radiology (A.G., R.J.Y., S.K., S.S., A.I.H.), Brain Tumor Center (R.J.Y., S.K., P.H.G., A.I.H., A.B.L.), Departments of Neurosurgery (P.H.G.), Epidemiology and Biostatistics (Z.Z., Q.M.), and Neurology (A.B.L.), Memorial Sloan-Kettering Cancer Center, New York, New York.

Ajay Gupta and Robert J. Young contributed equally as first authors.

Andrei I. Holodny and Andrew B. Lassman contributed equally as senior authors.

Paper previously presented at: Annual Meeting of the American Society of Neuroradiology, May 16-21, 2009; Vancouver, British Columbia, Canada.

Please address correspondence to Robert J. Young, MD, Department of Radiology, Memorial Sloan-Kettering Cancer Center, 1275 York Ave, MRI-1156, New York, NY 10065; e-mail: youngr@mskcc.org

DOI 10.3174/ajnr.A2479 explored, ${ }^{5,6}$ diffusion signatures are not commonly implemented into treatment management decision-making trees, and the significance of new restricted diffusion during treatment is unknown. Finding early imaging biomarkers for glioblastoma progression may have a significant impact on clinical treatment decision-making, especially during antiangiogenic therapy (eg, bevacizumab) that affects the blood-brain barrier and reduces the value of contrast enhancement as a technique for monitoring treatment response. Others have suggested restricted diffusion as a surrogate for disease progression in patients receiving bevacizumab ${ }^{7-11}$ or other antiangiogenic therapy. ${ }^{12}$

We hypothesized that diffusion restriction, when present outside of the confines of enhancing tumor, may be a useful predictor of disease progression. Therefore, our purpose was to determine the utility of diffusion restriction as a predictor of enhancing progression during treatment in all patients, both those receiving antiangiogenic therapy and those who did not. In addition, we sought to assess patient survival following the appearance of restricted diffusion during the course of treatment for glioblastoma.

\section{Materials and Methods}

\section{Patients and Follow-Up}

This retrospective study was granted a Waiver of Informed Consent by the hospital institutional review board. In compliance with HIPPA 


\begin{tabular}{lc}
\hline \multicolumn{2}{l}{ Table 1: Patient characteristics at time of first appearance of low- } \\
ADC lesion \\
\hline Patient Characteristics & No. \\
\hline Patients with low-ADC lesions & 27 \\
Age (median) (range) & $53(34-74)$ \\
Sex & \\
Men & 20 \\
Women & 7 \\
Chemotherapy at time of low-ADC lesion & \\
Contains bevacizumab & 15 \\
Bevacizumab only & 7 \\
Bevacizumab and irinotecan & 1 \\
Bevacizumab and temozolomide & 5 \\
Bevacizumab and carboplatin & 1 \\
Bevacizumab and lomustine & 1 \\
Does not contain bevacizumab & 6 \\
Temozolomide only & 6 \\
None & 6 \\
\hline
\end{tabular}

regulations, we identified from departmental data bases 208 patients diagnosed with histologically proved glioblastoma imaged from January 2005 to March 2010.

Review of all the MR imaging studies in these 208 patients revealed that $67(32.2 \%)$ had diffusion-restricted lesions manifest by high signal intensity on DWI and low signal intensity on ADC maps. The following criteria were applied to these 67 patients to determine the final study cohort: 1) no corresponding enhancement or diffusion restriction larger than enhancement, 2) no corresponding hemorrhage, 3) no immediate postoperative changes (ie, lesions at the margin of the surgical cavity on the immediate postoperative MR imaging were excluded), and 4) no corresponding clinical acute/subacute ischemia to explain the low-ADC lesion (as confirmed by the absence of clinical signs or symptoms consistent with ischemic stroke and the absence of gliosis or chronic infarction at follow-up). In addition, new enhancement was scored only if it occurred at the site of (concordant with) the low-ADC lesion. If multiple low-ADC lesions were present in a patient, only the first low-ADC lesion to develop enhancement was measured. Chart review was performed by a board-certified neuro-oncologist (A.B.L.) to confirm clinical details. A total of 27 patients met all inclusion criteria to form the study cohort.
There were 20 men and 7 women, with a median age of 53 years (range, 34-74 years).

If a patient developed new enhancement concordant with the low$\mathrm{ADC}$ lesion, all available advanced imaging (such as DSC T2* MR perfusion, DCE T1 MR perfusion, 3D multivoxel PROBE chemical shift imaging MR spectroscopy [GE Healthcare, Milwaukee, Wisconsin]) was reviewed by a board-certified neuroradiologist (with 10 years of experience) to determine the etiology of that new enhancement. The advanced imaging results were interpreted as representing tumor on the basis of the following criteria commonly applied at our institution and in the literature: $\mathrm{CCBV} \geq 1.75$ (on DSC MR perfusion), maximal bolus wash-in slope $\geq 2$ (on DCE MR perfusion), ${ }^{13,14}$ or Cho/NAA $\geq 2.2$ (MR spectroscopy). ${ }^{15-17}$

\section{MR Imaging and Diffusion Postprocessing}

Patients were imaged on 1.5T or 3T magnets (Signa HDx and Excite, GE Healthcare) by using standard quadrature head coils. Standard doses of $0.1-\mathrm{mmol} / \mathrm{kg}$ gadodiamide (Omniscan; Winthrop Laboratories, Rensselaer, New York) were used for the contrast-enhanced images. All studies were performed according to a standardized protocol that included DWI, ADC maps, and triplane contrast T1-weighted images. DWI was acquired by using single-shot echo-planar imaging with $8000 \mathrm{~ms}$ TR, $100 \mathrm{~ms}$ TE, $220-\mathrm{mm}$ FOV, $128 \times 128$ matrix size, $5-\mathrm{mm}$ section thickness with $0-2.5 \mathrm{~mm}$ intersection gap, and 1000 and $0 \mathrm{~mm}^{2} / \mathrm{s}$ b-values obtained in 3 orthogonal directions. Per institutional standard, patients were imaged approximately 1 month after completing radiation therapy and every 2 months thereafter.

\section{Image Interpretation}

Two radiologists with 4 years of experience in neuroradiology (A.G., S.S.), and 2 board-certified neuroradiologists holding Certificates of Added Qualification with 10 and 20 years of experience (R.J.Y., A.I.H.), and 1 board-certified neuro-oncologist with 9 years of experience (A.B.L.) interpreted the images in consensus while blinded to the clinical status and outcome. ADC measurements were obtained (by A.G., S.S.) by using region-of-interest analysis (Functools 4.1, Advantage Workstation, GE Healthcare). Quantitative ADC values were obtained by placing a region of interest (approximately $0.5 \mathrm{~cm}^{2}$
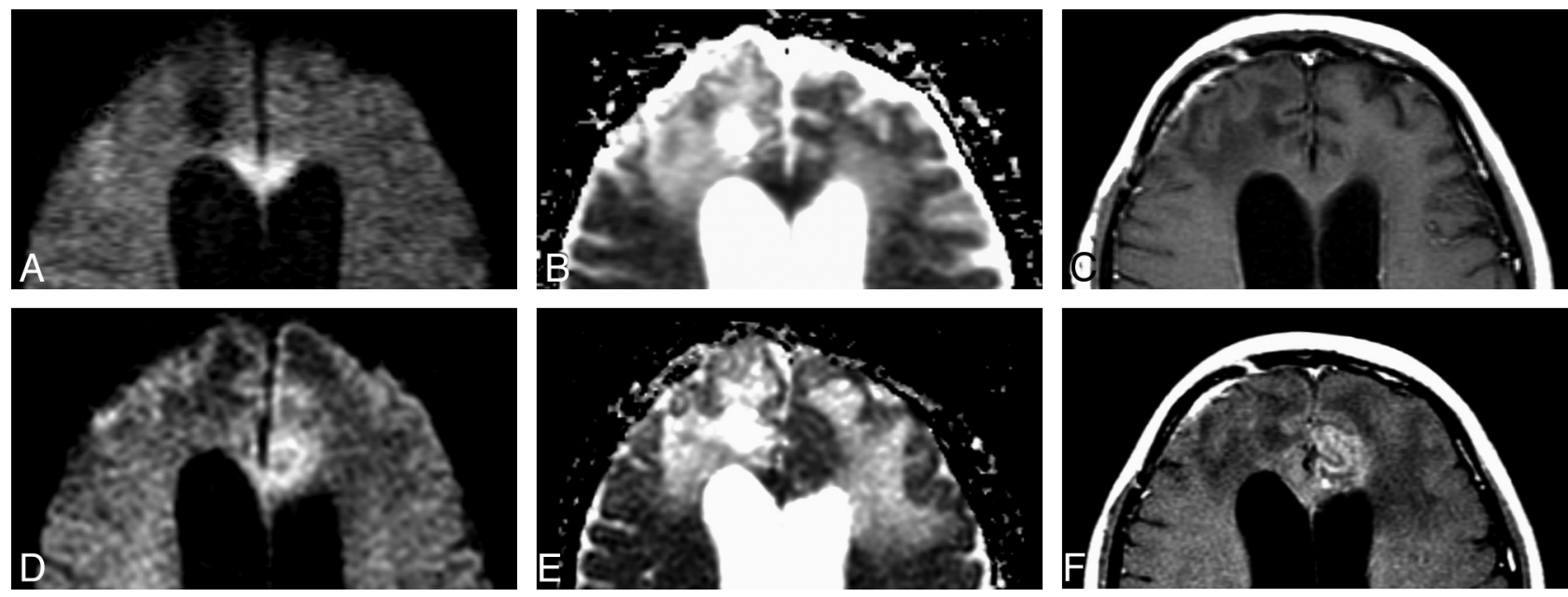

Fig 1. Representative low-ADC lesion in a patient with glioblastoma. $A-C$, Seven months after presentation (top row), there is a new recurrent lesion in the genu of the corpus callosum that has high signal intensity on DWI $(A)$, low signal intensity on $\operatorname{ADC}(B)$, and does not enhance on contrast-enhanced T1-weighted imaging $(C)$. Note associated postoperative and postradiation changes in the adjacent right frontal subcortical white matter. $D-F$, Five months later (bottom row), the lesion has increased in size and now also involves the left cingulate gyrus on DWI $(D)$ and $A D C(E)$ and shows new heterogeneous enhancement on contrast-enhanced T1-weighted imaging $(F)$. The enhancing lesion continued to progress on subsequent imaging performed approximately 1.5 months after figures $D-F$, and the patient died approximately 3 months later from tumor progression. 


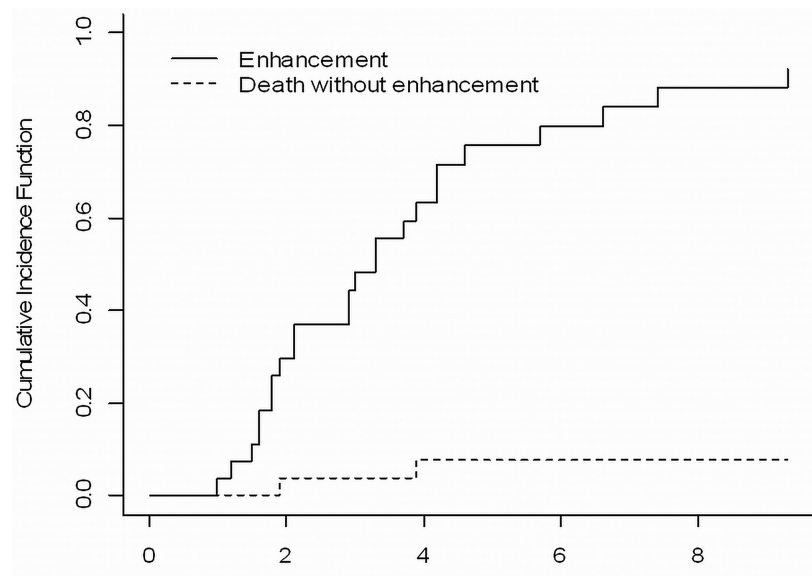

A

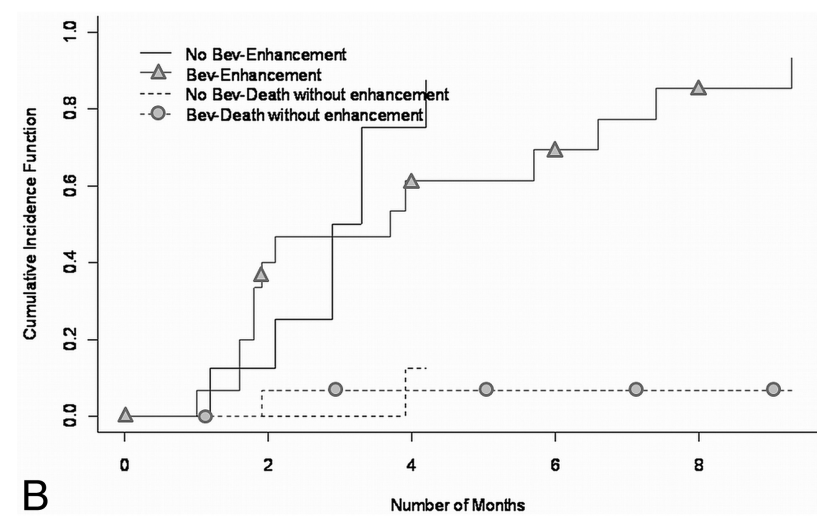

Fig 2. Enhancement-free survival calculated by using competing risk analysis for all patients $(A)$ and stratified by bevacizumab status $(B)$.

or 15 pixels) in the same anatomic region on every scan in the center of the lesion that would develop or had developed restricted diffusion. A minimum of 4 regions of interest was drawn for each measurement, adapted from a technique recommended by Wetzel et al, ${ }^{18}$ and the minimum value was recorded for ADC. On the follow-up studies, the development of enhancement within the region of restricted diffusion was assessed.

\section{Statistical Analysis}

The enhancement-free survival was determined by using competing risk analysis (because death without enhancement was regarded as a competing risk to development of enhancement). Overall survival was estimated by the Kaplan-Meier method. The enhancement-free and overall survival analyses were performed for all patients and then were stratified by treatment with or without bevacizumab during the first appearance of new low-ADC lesions. Gray and logrank tests were used to compare the probability of enhancement-free survival and overall survival between the bevacizumab and nonbevacizumab groups. The means of the ADC values obtained before and after the development of the low-ADC lesion were compared by using a Wilcoxon signed rank test. A Cox regression analysis was used to assess whether the magnitude of new diffusion restriction was correlated to the enhancement-free survival and overall survival.

\section{Results}

The demographic, clinical, and treatment characteristics of the 27 patients are summarized in Table 1. The low-ADC lesion occurred in 19 patients with newly diagnosed glioblas-
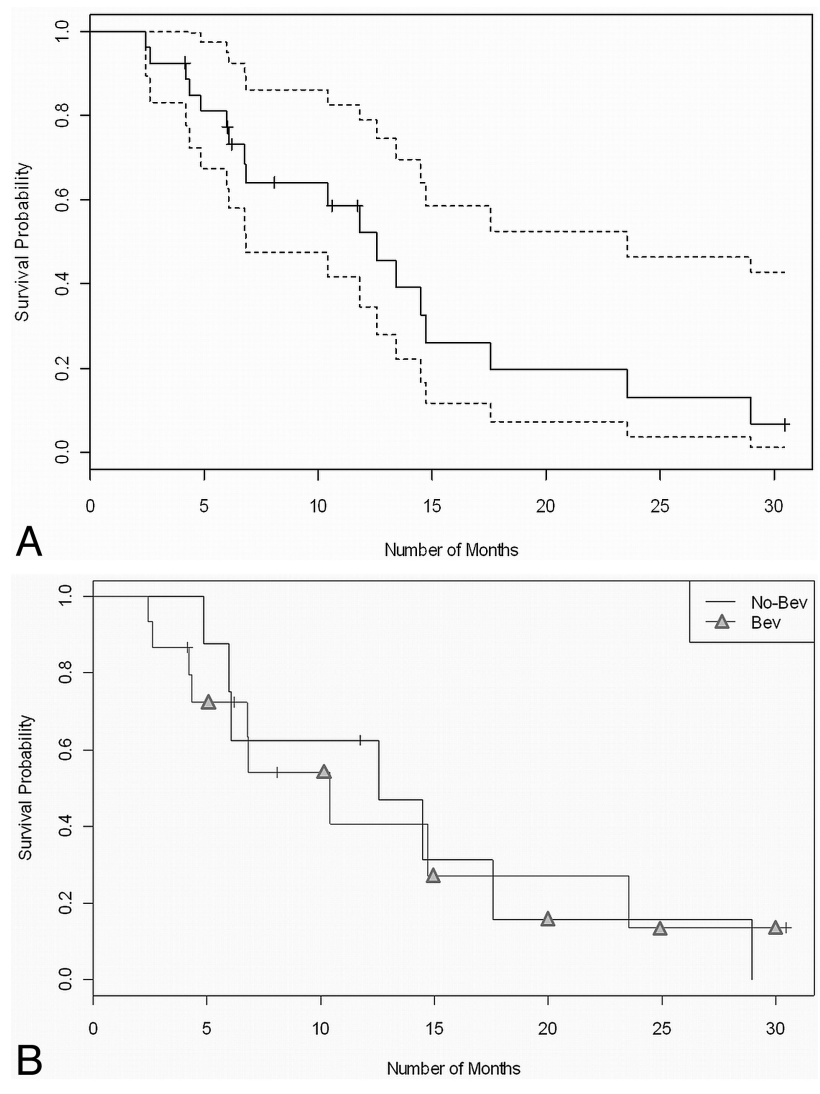

Fig 3. Overall survival calculated by using Kaplan-Meier curves for all patients $(A)$ and stratified by bevacizumab status $(B)$.

tomas and in 8 patients with recurrent disease and was always located in areas of T2 hyperintensity related to the tumor. Enhancement concordant with the low-ADC lesion $(n=23)$ or death before enhancement $(n=3)$ occurred in 26 of the 27 patients (96.3\%); only 1 patient is alive without enhancement. A representative case is shown in Fig 1. Median enhancementfree survival (Fig 2) was 3.0 months (95\% CI, 2.6-4.1 months). The 3-month enhancement-free survival probability was 0.481 (95\% CI, 0.288-0.675). Restricted diffusion predicted contrast enhancement regardless of treatment with bevacizumab $(P=.48)$. Median overall survival from the appearance of restricted diffusion (Fig 3) was 8.1 months (95\% CI, 9.7-11.2 months). The 12-month survival probability was 0.521 (95\% CI, 0.345-0.788). No statistically significant difference was found in overall survival between the bevacizumab and nonbevacizumab groups $(P=.97)$.

The mean decrease in ADC from baseline was $22.9 \%$ to 0.71 from $1.03 \times 10^{-5} \mathrm{~mm}^{2} / \mathrm{s}(n=20, P<.001)$. The lowADC lesions had a range of $0.44-0.97 \times 10^{-5} \mathrm{~mm}^{2} / \mathrm{s}$ with an

\begin{tabular}{ll}
\hline Table 2: Anatomic distribution of low-ADC lesions & \\
\hline Location & No. (\%) \\
\hline White matter tracts & $26(96.3)$ \\
Corpus callosum & 12 \\
Corona radiata & 4 \\
Centrum semiovale & 1 \\
Internal capsule & 2 \\
Other & 7 \\
Gray matter & 1 \\
Thalamic nucleus & $1(3.7)$ \\
\hline
\end{tabular}



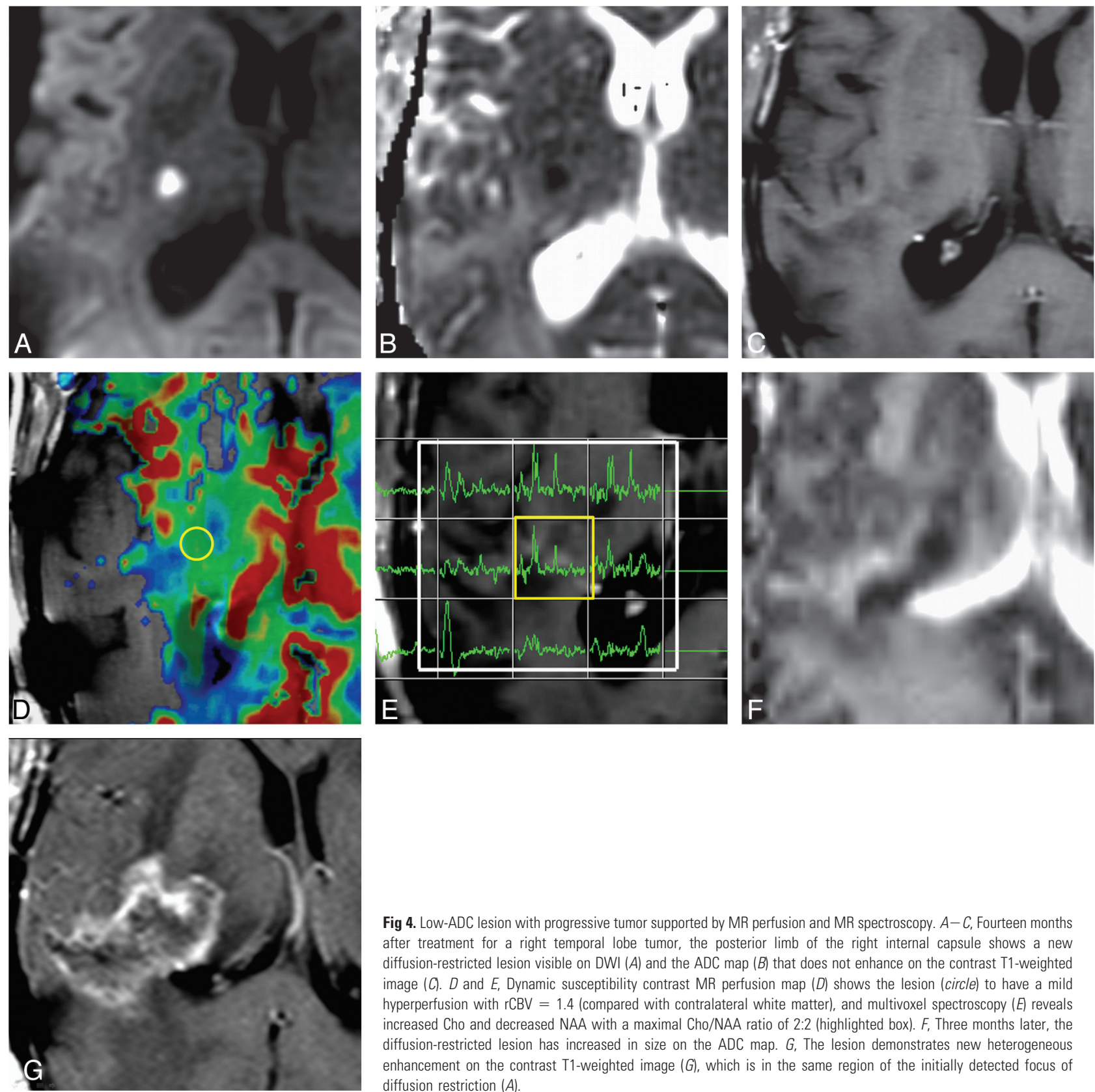

Fig 4. Low-ADC lesion with progressive tumor supported by MR perfusion and MR spectroscopy. $A-C$, Fourteen months after treatment for a right temporal lobe tumor, the posterior limb of the right internal capsule shows a new diffusion-restricted lesion visible on DWI $(A)$ and the ADC map $(B)$ that does not enhance on the contrast T1-weighted image (C). $D$ and $E$, Dynamic susceptibility contrast MR perfusion map (D) shows the lesion (circle) to have a mild hyperperfusion with rCBV $=1.4$ (compared with contralateral white matter), and multivoxel spectroscopy ( $E$ ) reveals increased Cho and decreased NAA with a maximal Cho/NAA ratio of 2:2 (highlighted box). F, Three months later, the diffusion-restricted lesion has increased in size on the ADC map. $G$, The lesion demonstrates new heterogeneous enhancement on the contrast T1-weighted image $(G)$, which is in the same region of the initially detected focus of diffusion restriction $(A)$.

$\mathrm{SD}$ of $0.13 \times 10^{-5} \mathrm{~mm}^{2} / \mathrm{s}$. No statistically significant correlation was detected between the magnitude of ADC decrease from baseline and the enhancement-free survival (HR, 0.542; $P=.75)$ or overall survival (HR, $0.03 ; P=.19$ ). The anatomic distribution of the low-ADC lesions is shown in Table 2. Twenty-six (96.3\%) low-ADC lesions were noted to extend along white matter tracts from the margin of the enhancing tumor.

All 27 patients received postoperative partial brain external beam radiation therapy designed by using a standard 2 - to $3-\mathrm{cm}$ margin around the resection cavity and/or residual enhancing mass lesion. The low-ADC lesions were all located within the radiation field. Twenty patients received standard radiation courses ( 60 Gy given as $2 \mathrm{~Gy} \times 30$ fractions for 6 weeks); 6 patients, hypofractionated radiation courses (36 Gy given as 6 Gy $\times 6$ fractions for 2 weeks, which is equivalent to standard 60 Gy given for 6 weeks); and 1 patient, an abbreviated radiation course to $41 \mathrm{~Gy}$ given as 2.76 Gy $\times 15$ fractions (an acceptable alternative for patients in poor condition). Twenty patients had completed radiation therapy before developing the low-ADC lesion with a median of 8.7 months; range, 0.9-188.0 months. The 23 patients who developed enhancement concordant with the low-ADC lesion had completed radiation therapy before the enhancement, with a median of 10.3 months (range, 0.5-192.1 months).

No patient had clinical signs or symptoms of acute/subacute ischemic stroke as an alternative explanation for restricted diffusion. Ultimately, all patients except 1 either clinically worsened or died from disease. Nine patients underwent advanced MR imaging of the new concordant enhancing lesions, with 3 patients undergoing $>1$ technique. Advanced MR imaging data in these patients were all consistent with recurrent tumor rather than treatment necrosis or ischemia: 
DSC MR perfusion imaging of the low-ADC lesion in 6 patients showed a mean rCBV of 2.1 (range, 0.9-2.9). An example is shown in Fig 4. When segregated by bevacizumab status, mean $\mathrm{rCBV}$ was 1.9 in patients $(n=5)$ receiving bevacizumab and 2.9 in the patient not receiving bevacizumab. DCE MR perfusion imaging in 1 patient showed a maximal bolus wash-in slope of 0.20 , maximal volume transfer constant $K^{\text {trans }}$ of $0.23 \mathrm{l} / \mathrm{min}$, and fractional volume of the extracellular extravascular space $\mathrm{V}_{\mathrm{e}}$ of 0.39 . In 2 patients, MR spectroscopy revealed increased mean Cho/NAA ratios of 3.3 (Fig 3).

\section{Discussion}

We found that a subset of patients with glioblastoma had isolated low-ADC lesions not explained by hemorrhage, ischemia, or postoperative change. These low-ADC lesions preceded new enhancing disease in the same location by a median of 3 months, with a 3-month enhancement-free survival probability of 0.481 . Like others, ${ }^{9-11}$ we found that restricted diffusion preceded enhancement in patients who were receiving bevacizumab. However, we also observed this phenomenon in patients who were not receiving antiangiogenic agents, suggesting restricted diffusion represents a true predictor of enhancement regardless of antiangiogenic treatment; further investigation in a larger patient population is warranted. Effort is underway to develop new response criteria in the treatment of glioblastomas, which recognize the imperfections associated with the exclusive reliance on measurement of contrastenhancing tumor size. ${ }^{19}$ Identifying $\mathrm{MR}$ imaging findings other than contrast enhancement to predict treatment response, particularly in patients receiving antiangiogenic agents, may provide significant clinical value. ${ }^{20}$

DWI is sensitive to alterations in the normal Brownian motion of water. Low ADC values have been correlated with increasing cellularity, increasing grade, and increasing Ki-67 cellular proliferation index in cerebral gliomas..$^{2-4,21-29} \mathrm{We}$ believe that the low ADC values reflect increased tumor cellularity with subsequent decreases in the free extracellular space and water proton diffusivity. It is also possible that relative tumor ischemia may contribute to the low ADC during a period of insufficient vascular proliferation and neovascularity that precedes abnormal enhancement and blood-brain barrier disruption. This concept of relative tumor ischemia is supported by pathologic work performed by Kleinschmidt-Demasters and Damek, ${ }^{30}$ who showed perivascular tumor deposits, vasculopathy, and small brain infarcts in bevacizumabtreated patients.

The anatomic distribution of the low-ADC lesions along white matter tracts is consistent with the well-described biologic dissemination of glioblastoma. ${ }^{31,32}$ These results are complemented by the work of Krishnan et al, ${ }^{33}$ who recently demonstrated that glioblastoma recurrences preferentially occurring along white matter tracts were visible on diffusion tensor imaging before they were visible on conventional imaging. In 11 of their 14 patients, tractography-generated fiber tracts could be seen leaving the site of the primary tumor and traveling to the site of the recurrent tumor. Diffusion and diffusion tensor imaging may become useful adjuncts in evaluating tumor progression when conventional imaging sequences yield normal results.

Several advanced imaging techniques have been proposed in the recent literature as earlier markers for tumor progression other than contrast enhancement. PET scans can be helpful, but the utility of the most commonly used radiotracer, fluorodeoxyglucose, is limited by the high preferential glucose uptake of normal brain, which reduces lesion-to-background conspicuity. This problem may be mitigated by some of the newer radiotracers, such as fluoro-L-thymidine ${ }^{34}$ and fluorocyclobutyl-carboxylic acid, ${ }^{35}$ under investigation at our center and others. MR perfusion and MR spectroscopy may also indicate tumor progression earlier than contrast enhancement. Although too few of the cohort patients in this study underwent these techniques to reach any significant conclusions, these techniques were helpful in confirming the enhancing lesions at the sites of initial low-ADC as progressive tumor. At this time, DWI is routinely performed as part of every brain study in all research and clinical settings, whereas MR perfusion, MR spectroscopy, and PET are not. Therefore, recognition of these low-ADC lesions has the widest potential impact on routine clinical care for patients with glioblastomas without requiring any additional imaging time, intravenous contrast, or imaging technique beyond standard practice.

One potential limitation is the relatively small patient cohort culled from the initial glioblastoma population. Despite the small numbers, we achieved statistically significant results that appear to define a low-ADC subgroup with biologic and imaging characteristics different from those of most glioblastomas. Another potential limitation relates to the different treatment protocols being used for our patients, with newly diagnosed as well as recurrent glioblastomas, which may alter the enhancement-free survival results. While the combination of concurrent radiation therapy and temozolomide is the current standard of care for glioblastomas, ${ }^{36}$ many of our patients had failed standard therapy and were on different investigational protocols, including the use of bevacizumab alone or in combination. The ability of the low-ADC lesions to predict enhancing disease appears to be maintained across different treatment protocols, however, and is a feature that may augment its potential utility in clinical care.

A third potential limitation is the lack of locus-specific histopathologic correlation in our series to confirm the new enhancing lesions at the site of the low-ADC lesions as tumor rather than pseudoprogression or radiation necrosis, because this was not clinically indicated for the reported patients. However, others have reported histologic proof of recurrent glioblastoma as the cause of restricted diffusion. ${ }^{11}$ In addition, advanced imaging of the concordant enhancement (MR perfusion or MR spectroscopy) corroborated the presence of tumor. Furthermore, patients in our series developed enhancement concordant with the low-ADC lesion after a median of 10.3 months. Although later development is possible, pseudoprogression typically occurs within the first 3 months after completing radiation therapy. Accordingly, the timing of enhancement in our cohort is more suggestive of tumor progression rather than treatment effect. ${ }^{37}$

Finally, the reported variability in ADC values among and even within vendors is another limitation of this study. ${ }^{38}$ However, all of our patients were scanned on MR imaging scanners from 1 vender. In terms of the ADC measurements, the degree of $\mathrm{ADC}$ decrease did not correlate with the enhancement-free survival. We found all low-ADC lesions to be 
visibly apparent on the DWI and ADC maps and clearly abnormal even without any quantitative measurements. Although obtaining a relative percentage decrease in ADC or normalized ADC value may be helpful, we believe that visual inspection is sufficient to determine the presence of diffusion restriction. The lack of correlation between the magnitude of decrease in ADC and enhancement-free survival or overall survival further supports the utility of simple qualitative evaluation.

\section{Conclusions}

Isolated low-ADC lesions in a subset of patients with glioblastoma precedes the development of concordant enhancing lesions at the same site. Larger prospective trials are needed to confirm our findings, but our preliminary data support the role of using low-ADC lesions as potential harbingers of tumor progression that should be included in the clinical treatment decision-making process, regardless of treatment with bevacizumab. Additional work is necessary to obtain histopathologic confirmation and to determine the potential impact on patient survival through targeted surgical or radiation therapy.

\section{Acknowledgments}

We thank Bob Hou, MD, for his generous assistance in DCE MR perfusion data analysis.

Disclosures: Zhigang Zhang. Research Support lincluding provision of equipment or materials): Memorial Sloan-Kettering Cancer Center; Andrew B. Lassman as senior authors. Research Support (including provision of equipment or materials): Genentech, Schering Plough (Merck), Sigma Tau; Speaker Bureau: Schering Plough; Details: Ended 12/31/2009; Consultant. see below in the past year; Details: Campu Bio 3/2010, Eisai 1/8/2010, Schering Plough 9/21/09, Schering Plough 9/11/09, Schering Plough 3/5/2010.

\section{References}

1. Louis DN, Ohgaki H, Wiestler OD, et al. The 2007 WHO classification of tumours of the central nervous system. Acta Neuropathol 2007;114:97-109. Epub 2007 Jul 6

2. Castillo M, Smith JK, Kwock L, et al. Apparent diffusion coefficients in the evaluation of high-grade cerebral gliomas. AJNR Am J Neuroradiol 2001;22:60-64

3. Guo AC, Cummings TJ, Dash RC, et al. Lymphomas and high-grade astrocytomas: comparison of water diffusibility and histologic characteristics. Radiology 2002;224:177-83

4. Higano S, Yun X, Kumabe T, et al. Malignant astrocytic tumors: clinical importance of apparent diffusion coefficient in prediction of grade and prognosis. Radiology 2006;241:839-46

5. Saksena S, Jain R, Narang J, et al. Predicting survival in glioblastomas using diffusion tensor imaging metrics. J Magn Reson Imaging 2010;32:788-95

6. Murakami R, Sugahara T, Nakamura H, et al. Malignant supratentorial astrocytoma treated with postoperative radiation therapy: prognostic value of pretreatment quantitative diffusion-weighted MR imaging. Radiology 2007;243:493-99

7. Jain R, Scarpace LM, Ellika S, et al. Imaging response criteria for recurrent gliomas treated with bevacizumab: role of diffusion-weighted imaging as an imaging biomarker. J Neurooncol 2010;96:423-31

8. Rieger J, Bahr O, Muller K, et al. Bevacizumab-induced diffusion-restricted lesions in malignant glioma patients. J Neurooncol 2010;99:49-56

9. Mohile NA, Abrey LE. Assessment of treatment response to bevacizumab (bev) with MRI in recurrent malignant glioma (MG) [abstract MA-05]. Neuro Oncol 2007;9:516

10. Pope WB, Kim HJ, Huo J, et al. Recurrent glioblastoma multiforme: ADC histogram analysis predicts response to bevacizumab treatment. Radiology 2009;252:182-89

11. Gerstner ER, Frosch MP, Batchelor TT. Diffusion magnetic resonance imaging detects pathologically confirmed, nonenhancing tumor progression in a patient with recurrent glioblastoma receiving bevacizumab. J Clin Oncol 2010;28:e91-93
12. Gerstner ER, Chen PJ, Wen PY, et al. Infiltrative patterns of glioblastoma spread detected via diffusion MRI after treatment with cediranib. Neuro Oncol 2010;12:466-72. Epub 2010 Jan 22

13. Gutin PH, Iwamoto FM, Beal K, et al. Safety and efficacy of bevacizumab with hypofractionated stereotactic irradiation for recurrent malignant gliomas. Int J Radiat Oncol Biol Phys 2009;75:156-63

14. Larsson HB, Tofts PS. Measurement of blood-brain barrier permeability using dynamic Gd-DTPA scanning: a comparison of methods. Magn Reson Med 1992;24:174-76

15. Law M, Oh S, Johnson G, et al. Perfusion magnetic resonance imaging predicts patient outcome as an adjunct to histopathology: a second reference standard in the surgical and nonsurgical treatment of low-grade gliomas. Neurosurgery 2006;58:1099-107, discussion 1099-107

16. Young RJ, Knopp EA. Brain MRI: tumor evaluation. J Magn Reson Imaging 2006;24:709-24

17. Al-Okaili RN, Krejza J, Wang S, et al. Advanced MR imaging techniques in the diagnosis of intraaxial brain tumors in adults. Radiographics 2006;26(suppl 1):S173-89

18. Wetzel SG, Cha S, Johnson G, et al. Relative cerebral blood volume measurements in intracranial mass lesions: interobserver and intraobserver reproducibility study. Radiology 2002;224:797-803

19. Wen PY, Macdonald DR, Reardon DA, et al. Updated response assessment criteria for high-grade gliomas: response assessment in neuro-oncology working group. J Clin Oncol 2010;28:1963-72. Epub 2010 Mar 15

20. Hormigo A, Gutin PH, Rafii S. Tracking normalization of brain tumor vasculature by magnetic imaging and proangiogenic biomarkers. Cancer Cell 2007;11:6-8

21. Sugahara T, Korogi Y, Kochi M, et al. Usefulness of diffusion-weighted MRI with echo-planar technique in the evaluation of cellularity in gliomas. J Magn Reson Imaging 1999;9:53-60

22. Gupta RK, Cloughesy TF, Sinha U, et al. Relationships between choline magnetic resonance spectroscopy, apparent diffusion coefficient and quantitative histopathology in human glioma. J Neurooncol 2000;50:215-26

23. Gupta RK, Sinha U, Cloughesy TF, et al. Inverse correlation between choline magnetic resonance spectroscopy signal intensity and the apparent diffusion coefficient in human glioma. Magn Reson Med 1999;41:2-7

24. Bulakbasi N, Guvenc I, Onguru O, et al. The added value of the apparent diffusion coefficient calculation to magnetic resonance imaging in the differentiation and grading of malignant brain tumors. J Comput Assist Tomogr 2004;28:735-46

25. Fan GG, Deng QL, Wu ZH, et al. Usefulness of diffusion/perfusion-weighted MRI in patients with non-enhancing supratentorial brain gliomas: a valuable tool to predict tumour grading? Br J Radiol 2006;79:652-58. Epub 2006 Apr 26

26. Kim HS, Kim SY. A prospective study on the added value of pulsed arterial spin-labeling and apparent diffusion coefficients in the grading of gliomas. AJNR Am J Neuroradiol 2007;28:1693-99

27. Kitis $\mathrm{O}$, Altay $\mathrm{H}$, Calli $\mathrm{C}$, et al. Minimum apparent diffusion coefficients in the evaluation of brain tumors. Eur J Radiol 2005;55:393-400

28. Tozer DJ, Jager HR, Danchaivijitr N, et al. Apparent diffusion coefficient histograms may predict low-grade glioma subtype. NMR Biomed 2007;20:49-57

29. Alvarez-Linera J, Benito-Leon J, Escribano J, et al. Predicting the histopathological grade of cerebral gliomas using high b value MR DW imaging at 3-Tesla. J Neuroimaging 2008;18:276-81. Epub 2008 May 5

30. Kleinschmidt-Demasters BK, Damek DM. The imaging and neuropathological effects of bevacizumab (Avastin) in patients with leptomeningeal carcinomatosis. J Neurooncol 2010;96:375-84. Epub 2009 Jul 16

31. Rees JH, Smirniotopoulos JG, Jones RV, et al. Glioblastoma multiforme: radiologic-pathologic correlation. Radiographics 1996;16:1413-38, quiz 1462-63

32. Scherer HJ. The forms of growth in gliomas and their practical significance. Brain 1940;63:1-35

33. Krishnan AP, Asher IM, Davis D, et al. Evidence that MR diffusion tensor imaging (tractography) predicts the natural history of regional progression in patients irradiated conformally for primary brain tumors. Int J Radiat Oncol Biol Phys 2008;71:1553-62. Epub 2008 Jun 4

34. Chen W, Delaloye S, Silverman DH, et al. Predicting treatment response of malignant gliomas to bevacizumab and irinotecan by imaging proliferation with $[18 \mathrm{~F}]$ fluorothymidine positron emission tomography: a pilot study. J Clin Oncol 2007;25:4714-21

35. Grommes C, Akhurst TJ, Blasberg RG, et al. [18F]FACBC and [18F]FLT PET imaging for glioblastomas (GBM) [abstract]. Neurology 2010; 74 (Suppl 2): A594

36. Stupp R, Mason WP, van den Bent MJ, et al. Radiotherapy plus concomitant and adjuvant temozolomide for glioblastoma. N Engl J Med 2005;352:987-96

37. Brandes AA, Tosoni A, Spagnolli F, et al. Disease progression or pseudoprogression after concomitant radiochemotherapy treatment: pitfalls in neurooncology. Neuro Oncol 2008;10:361-67

38. Sasaki M, Yamada K, Watanabe Y, et al. Variability in absolute apparent diffusion coefficient values across different platforms may be substantial: a multivendor, multi-institutional comparison study. Radiology 2008;249:624-30 